\title{
One organ at a time
}

\author{
Research has been making much progress to create in vitro human tissues for transplantation but \\ laboratory-grown complex organs still remain decades away
}

Philip Hunter

S tem cell research-and investment into it-has been largely inspired by the hope of using stem cells to regenerate, repair or replace damaged tissue or even whole organs. Stem cell-based therapies promise to eventually solve the problems associated with conventional organ transplantation: notably tissue rejection, a shortage of donors and the poor quality of donor organs. A much improved knowledge of the factors needed to coax stem cells to differentiate into specialized cells, in combination with advances in tissue engineering, has brought that promise closer to reality. Specific tissues and even whole organs generated from stem cells can now be grown in vitro and then transplanted into a patient to continue their development in vivo. Scientists have already successfully transplanted relatively simple laboratory-grown organs, such as the bladder and wind-pipe, and have for decades conducted now-standard skin and bone marrow transplantation.

"Specific tissues and even whole organs generated from stem cells can now be grown in vitro and then transplanted into a patient to continue their development in vivo"

The first laboratory-grown internal organs were transplanted in 1999 in the USA, although the results were not reported until 2006, after tracking the patients for several years [1]. These first transplants did not involve the specific differentiation of stem cells, but they established that laboratory-grown organs could be transplanted and subsequently function correctly. The team involved, under the leadership of Anthony Atala, Director of the Wake Forest Institute for Regenerative Medicine in Winston-Salem, North Carolina, implanted laboratory-grown bladders into seven children with spina bifida and severely malfunctioning bladders. They extracted cells from the children's malfunctioning bladders and used them to grow thin sacs of tissue in the laboratory. These rudimentary organs were then grafted back on to the patients' own bladders. The work was an important stepping stone and highlighted the need, at least for the time being, for some form of backbone or scaffold to provide the cues needed for cells to organize themselves into appropriate three-dimensional structures while differentiating.

The first transplant of an organ that was specifically generated from a patient's own stem cells came almost a decade later at University College, London, UK. A team led by Martin Birchall, professor of Laryngology at the UK's Royal National Throat, Nose and Ear Hospital, successfully grew a trachea from the patient's stem cells and transplanted it back into the patient [2; Fig 1]. This achievement was followed 2 years later by performing the same procedure on a boy, who became the first child to receive a stem cell-engineered organ, by the same team. "The boy is growing normally and is at school," Birchall confirmed.

These procedures also pioneered the use of a scaffold on which to implant the stem cells; Birchall's team used the fibrous collagen from a donor trachea. To avoid any problems with rejection, the scaffold was stripped of the donor cells to make sure that only the patient's own cells were implanted. Birchall says his team have since performed four more stem cell-generated trachea transplants, including one using an artificial scaffold to avoid the need for a donor organ.

ए rom the perspective of in vitro generation and transplantation, organs come in four categories of increasing complexity, according to Atala, who is still working on bladder transplants and has expanded his work into other solid organs. "Firstly flat structures, such as skin, are the easiest to engineer and skin was the first 'organ' to be engineered in the laboratory," he said. This category also includes bone marrow, which was first transplanted in 1968 to treat an infant suffering from $\mathrm{X}$-linked lymphopenic immune deficiency. This early procedure involved a whole marrow, but more recent treatments have just transplanted bone marrow stem cells.

"Among the solid organs, the liver is generally regarded as the most promising target for regenerative medicine"

"Then tubular structures, such as blood vessels and the windpipe, are next in complexity," Atala continued. "Thirdly, hollow organs, such as the bladder and stomach, are even more complex because, unlike simple conduits, they must work in concert

Freelance journalist in London, UK.

DOI 10.1002/embr.201438528 | Published online 19 February 2014 


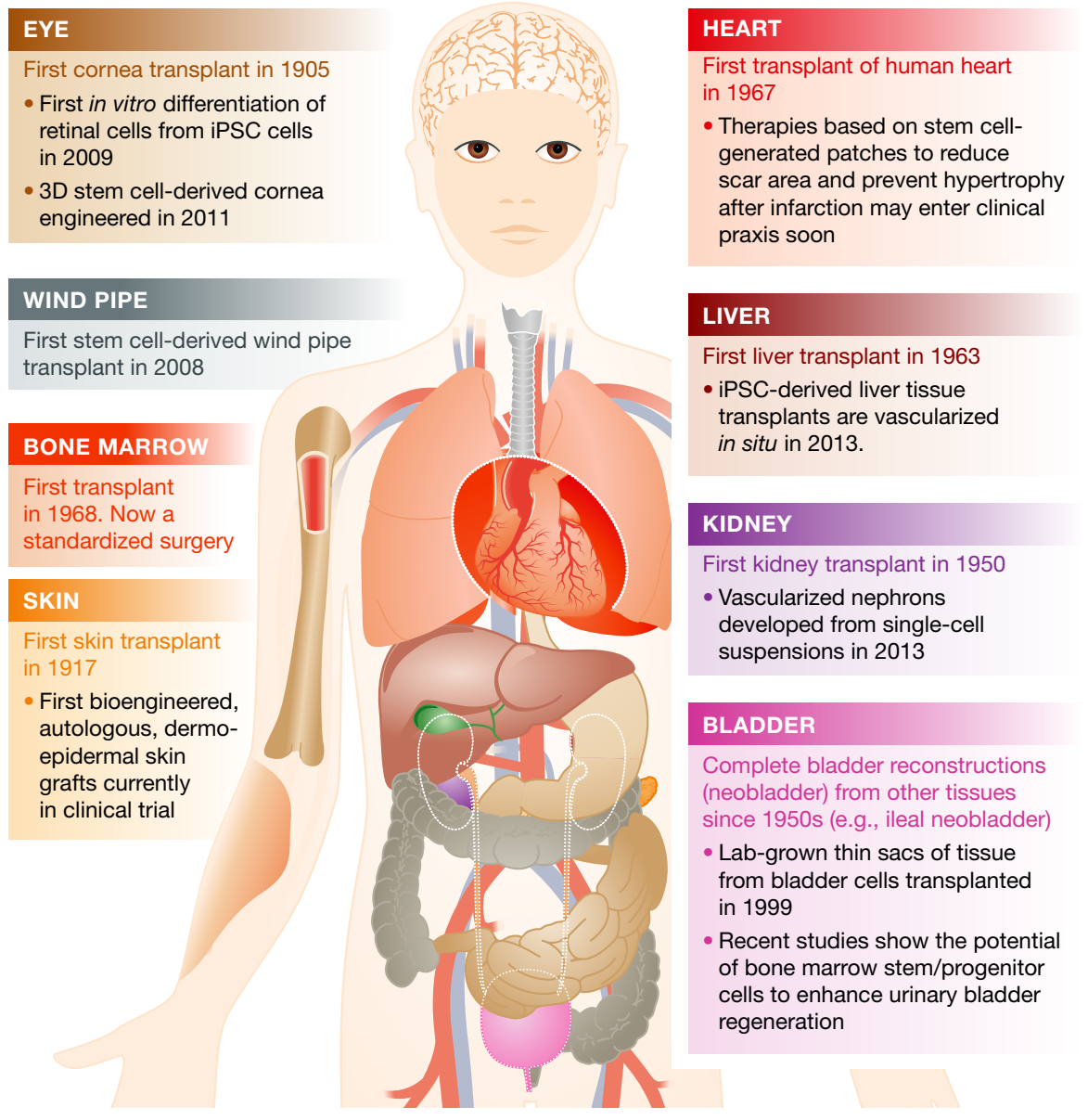

Fig 1. Organ transplantations and tissue and organ engineering.
A team at Yokohama City University Graduate School of Medicine in Japan, led by Takanori Takebe, Associate Professor in the Department of Regenerative Medicine, has made further progress on generating liver tissue on two fronts. "Firstly, we have established a large-scale 3D tissue selforganizing culture system without the aid of conventional scaffolds, enabling multiple cellular types to interact in a spatiotemporal manner as observed in organogenesis," explained Takebe. "Second, we employed a new concept of iPSC differentiation, enabling the spatiotemporal regulation of multicellular communication in iPSCs to derive fully functioning cells." Takebe and colleagues have thus come closer to emulating in vivo organogenesis, which depends on molecular cues governed by the relative locations of differentiating and multiplying cells at a given point in time. Moreover, their approach has overcome another crucial hurdle: hooking up implanted tissue to the host's vascular system so that it obtains the nutrients needed for growth and development. Takebe's team found that less than $48 \mathrm{~h}$ after transplantation into mice, the 3D tissue created in a petri dish without a scaffold had gained access to the host's blood supply.

S oto-Gutierrez believes that developments like these foster further collaboration to push towards creating a fully functional stem cell-derived liver. "The next step is to combine technology derived from stem cells and cell biology with transplantation and bioengineering knowledge and make a human liver," he said. "The techniques are there now and we just need to get them together." He added that these techniques are also applicable to tackle other, more complex organs. "The second target will be the lung, because you can still transplant one lung and have the other functional," he said. The third, he thinks, will be the heart, although not initially as a whole organ, but more likely a supplementary pump to provide assistance. The largest challenge, he said, will be to grow kidney transplants, because the organ is more complex and requires close cooperation between several cell types. At the same time, there is increasing demand for kidney transplantation because kidney disease is a growing problem for many countries as a result of longevity and lifestyle factors. According to the 2010 Global 
Burden of Disease study, chronic kidney disease was ranked $27^{\text {th }}$ in the list of causes of total number of global deaths in 1990, but had risen to 18 th in 2010 at a rate of 16.3 per 100,000 [3].

\section{"The liver is also easier to transplant because only pieces rather than the whole organ are required"}

As with the liver, there are two halves to the problem of growing a kidney. Jamie Davies at the Centre for Integrative Physiology at Edinburgh University in the UK is tackling the first half: tissue engineering to produce working kidneys from renal stem cells. His team has shown that nephrons generated from single-cell suspensions can be implanted and vascularized, which Davies indicated is an important step towards replacing full renal function with a tissue-engineered kidney. "Working with Italian colleagues who have expertise with in vivo work, we have shown that engineered kidneys will hook up to host blood supplies and will filter blood," he said. "We are currently making detailed physiological measurements of the function of our engineered kidneys, but as the work has not yet been accepted for publication [...], I should not make any specific claims about this.” Each kidney has around one million nephrons, which are both structural and functional units, performing the filtering and various regulatory functions of the kidney, including the concentration of urine and regulation of blood pressure. Davies added that progress is also being made to include a ureter in the engineered kidneys, but this task had proved unexpectedly challenging. "Clearly the urine has to get out, but this apparently trivial feature is actually awkward to build in because we have to stop the rest of the developing kidney persuading the ureter to become yet more collecting duct."

Davies noted that other teams have been making equally striking progress on generating the required human renal stem cells from either hESCs or iPSCs. "There has been steady progress in designing the right witch's brew of growth factors, generally designed to recapitulate the normal developmental sequence," he said. He cited a recently pub- lished Australian study as an example, but also warned against overstating progress [4]. "What this [publication] actually achieves is cells making some little cysty things in $3 \mathrm{D}$ culture that express antigens characteristic of developing nephrons," Davies explained. "This is good work, but calling what was achieved a 'self-organizing kidney' is going to raise unrealistic public expectations. That kind of thing always ends up coming back to damage a field of research, as we are seen not to deliver what we scientists in general seem to have promised."

$t$ is really the title of the Australian paper that is over-ambitious, rather than the content and discussion in the study itself. The paper's lead author, Melissa Little from the University of Queensland, agrees that much more research is needed to proceed to a functional kidney in vitro. "We need to show evidence of further differentiation and examine scale up," she said. "What we show is an ability to begin the formation of a developing kidney. The most important aspect of our study is that we have been able to simultaneously induce multiple renal progenitor subtypes and show that they can induce each other to form renal tubules. Hence, we can begin to get structures similar to the developing embryonic kidneys from explants containing cells all derived from human pluripotent cells."

The study was performed using predominantly hESCs, but the supplementary data indicate in principle how the technique could be transferred to iPSCs, which would enable developing kidneys to match individual patients in the distant future. "In theory, the approach could be applied to iPSC lines taken from specific patients," Little explained. "However, this technology is far from providing a cellular therapy and may be much closer to use in drug testing."

A lthough it is technically not "solid," the eye is another complex organ that is becoming the focus of stem cell regeneration therapies. It is also highly challenging, as it rivals or even exceeds some solid organs in terms of complexity: it contains vitreous humour, giving it a $3 \mathrm{D}$ structure, and a range of highly specialized epithelial, receptor and neuron cells within an exactly defined architecture. While whole eyes are rarely needed, key components such as the cornea, the lens or the retina are interesting targets for transplantation. Thus, the 2009 publication by a Japanese team at the RIKEN Center for Developmental Biology in Kobe about the first in vitro differentiation of iPSCs into retinal progenitors, retinal pigment epithelium cells and photoreceptors, heralded a milestone in generating crucial cell types for these tissues [5].

\section{"The largest challenge, he said, will be to grow kidney transplants, because the organ is more complex and requires close cooperation between several cell types"}

The other solid organ that is attracting a lot of attention is the heart, given its long history of transplantation that dates back to Christian Barnard's famous operation in South Africa in 1967. Since then, heart transplants have become relatively routine and now exceed 4,000 a year worldwide. Prognosis has also improved steadily, with the median survival time now at 11 years (http://www.ishlt.org/registries/slides.asp? slides = heartLungRegistry). But the donor hearts available are both limited in number and tend to have come from older people, and so are not in pristine condition. As such, stem cell-derived heart tissues could alleviate the shortage and quality issues, not necessarily as complete hearts, but rather as components or patches to improve the functionality of diseased hearts as an alternative to transplantation.

A Spanish team led by Francisco FernandezAviles, Head of Cardiology at Hospital General Universitario Gregorio Marañon in Madrid, has been testing the use of stem cells to generate tissues that can be used to patch up damaged heart muscle after disease or infarction. "Specifically, we are developing preclinical studies in pigs in which cardiac decellularized tissues are repopulated with different species of stem cells and placed on top of the infarct area," he explained. "This technology is expected to reduce the scar area and prevent the development of hypertrophy [thickening of the ventricular chamber walls] that decreases the efficiency of the patient's heart."

Therapies based on such patches will soon be ready to enter clinical practice, FernandezAviles suggested, although more research is 
needed to make them work optimally in synchronization with the rest of the patient's heart: heart muscle cells need to act in harmony to efficiently fulfil their function of pumping blood. "Whereas in a liver or a lung each individual cell acts almost independently, in the heart there is a strong interaction between cells," Fernandez-Aviles said.

"... donor hearts available are both limited in number and tend to have come from older people, and so are not in pristine condition"

"In fact, only the synergy produced by the electrical and mechanical communication between cardiomyocytes (heart muscle cells) can produce an effective contraction of the heart. This explains why, while a few hepatocytes can reproduce almost all functions of an adult liver, a whole heart is needed to achieve a functional blood ejection."

To tackle this problem and develop cardiac parches that accurately mimic the electromechanical behaviour of a patient's heart, Fernandez-Aviles and his team have developed a vest that allows them to map the patient's heart's electrical activity so as to identify scar tissue. "This system allows the characterization of the electrical activity of a patient faster than through insertion of a catheter, helping in the diagnostic and in the planning of the intervention," he explained. Although it will still be several decades before whole hearts can be generated from a patient's stem cells, FernandezAviles believes that partial replacement via patches is imminent and will make an increasing impact in the clinic, given a continuing shortage of donor organs.

In contrast, Soto-Gutierrez thinks that a complete laboratory-generated liver could become available within as little as a decade, in part because of the organ's inherent regenerative capability. Given the current rate of progress in stem cell research and three-dimensional tissue engineering, this may not be an exaggerated prospect.

\section{Conflict of interest}

The author declares that he has no conflict of interest.

\section{References}

1. Atala A, Bauer SB, Soker S, Yoo JJ, Retik AB (2006) Tissue-engineered autologous bladders for patients needing cystoplasty. Lancet 367 : $1241-1246$

2. Hollander A, Macchiarini P, Gordijn B, Birchall M (2009) The first stem cell-based tissue-engineered organ replacement: implications for regenerative medicine and society. Regen Med 4: $147-148$

3. Lozano R, Naghavi M, Foreman K, Lim S, Shibuya K, Aboyans V, Abraham J, Adair T, Aggarwal R, Ahn SY (2013) Global and regional mortality from 235 causes of death for 20 age groups in 1990 and 2010: a systematic analysis for the Global Burden of Disease Study 2010. Lancet 380: 2095-2128

4. Takasato M, Er PX, Becroft M, Vanslambrouck JM, Stanley EG, Elefanty AG, Little MH (2014) Directing human embryonic stem cell differentiation towards a renal lineage generates a self-organizing kidney. Nat Cell Biol 16: $118-126$

5. Osakada F, Jin ZB, Hirami Y, Ikeda H, Danjyo T, Watanabe K, Sasai Y, Takahashi M (2009) In vitro differentiation of retinal cells from human pluripotent stem cells by small-molecule induction. J Cell Sci 122: $3169-3179$ 